IOS Press

\title{
Home-based rehabilitation in patients over 60 with stabilized ankylosing spondylitis during the COVID-19 pandemic
}

\author{
Kyunghwan $\mathrm{Cho}^{\mathrm{a}}$ and Austin Kang ${ }^{\mathrm{b}, *}$ \\ ${ }^{a}$ Department of Health Rehabilitation, Hanshin University, Korea \\ ${ }^{\mathrm{b}}$ Department of Forensic Medicine, Seoul National University, Korea
}

Received 2 June 2021

Accepted 11 October 2021

\begin{abstract}
.
BACKGROUND: COVID-19 has become a significant healthcare issue, particularly challenging for patients with ankylosing spondylitis (AS), because immune-related diseases and their treatments could adversely affect the susceptibility to or severity of a viral infection.

OBJECTIVE: This study is conducted to present an exercise rehabilitation program that patients older than 60 years with AS can do at home during the COVID-19 pandemic.

METHODS: Three Delphi surveys were conducted to reach a consensus on home-based rehabilitation programs. This study recruited ten experts and performed three Delphi rounds for a month.

RESULTS: The expert panel suggested that home-based rehabilitation for the patients should be carried out with a clear rehabilitation goal. Their final recommendations are to institute a program aimed to ease symptoms, such as pain and stiffness; encourage patients to consult with experts regularly to ensure that they perform exercise rehabilitation properly at home; add fast walking and stretching to the rehabilitation program; and see if indoor cycling, Pilates, or yoga could be appropriate.

CONCLUSIONS: This study suggests that patients with AS over 60 should repeat low-intensity exercises, such as stretching, for an hour a day, four to six times a week during the COVID-19 pandemic.
\end{abstract}

Keywords: Ankylosing spondylitis, home-based rehabilitation, COVID-19

\section{Introduction}

The COVID-19 pandemic has become a significant healthcare issue worldwide and is particularly challenging for patients with ankylosing spondylitis (AS), because immune-related diseases and their treatments could adversely affect the susceptibility to or severity of a viral infection [1,2]. The medical consensus regarding health risks related to the COVID-19 pandemic is that

${ }^{*}$ Corresponding author: Austin Kang, Department of Forensic Medicine, Seoul National University, Korea. E-mail: animus70@snu. ac.kr. the elderly are by far the most vulnerable population. Age is the most important factor in diminishing the chances of surviving COVID-19, individuals over the age of 65 are particularly vulnerable [3]. In the absence of effective pharmacological intervention, managing the virus largely depends upon effective public health measures to mitigate its spread and flatten the curve [4]. These measures have been implemented in many countries and include bans on public gatherings, compulsory stay-at-home policies, required closure of schools and nonessential businesses, and ordinances requiring face masks, quarantines, and cordon sanitaire $[5,6]$.

Patients with AS often experience pain and stiffness in their joints, which can be treated with rehabilitation 
exercises. Regular exercise is recommended to prevent the disease's progression, and remains necessary during the COVID-19 pandemic to mitigate symptoms [7]. However, hospital visits may increase the patient's risk of infection. Home-based rehabilitation is an alternative support system, which can allow care to continue in a familiar environment, reducing the burden on caregivers and improving the subject's quality of life [8].

Several studies indicate that home rehabilitation can be as effective as outpatient clinics, with no significant differences between pediatric and adult patients' outcomes [9]. Nevertheless, developing effective homebased exercise routines for the rehabilitation of patients with AS in their $60 \mathrm{~s}$ or older remains a significant task. It is a multifactorial problem, confronting situational and psychological factors that vary between individuals, and that need to be addressed by experts in the design of personalized exercise programs [10]. Azhari and Parsa [11] reported the importance of home rehabilitation in orthopedic areas during the COVID-19 pandemic. According to them, when social distancing policies limit hospital or clinic-based rehabilitation, home-based rehabilitation can be one of the supportive programs that allow the continuation of patient care in a familiar living environment to reduce the burden on caregivers and improve the quality of life of the patients. However, their report was a letter to the editor, with the limitation that no empirical analysis was presented on how to implement home-based rehabilitation programs for patients with stabilized AS during the COVID-19 pandemic. Thus, the exercise programs that experts identify as being the most effective in maintaining joint flexibility and relaxing rigid joints and muscles should be accessible to patients with AS without expert assistance. The purpose of this study was to present an exercise rehabilitation program for older patients with AS to do at home during the COVID-19 pandemic.

\section{Literature review}

Ankylosing spondylitis is a chronic inflammatory disorder that mainly affects sacroiliac joints and spine joints, causing severe, chronic pain [12]. Excessive inflammation can result in local tissue damage, and progressive joint immobility, leading to spinal deformities [13]. The current treatments for AS include medications with non-steroidalal anti-inflammatory drugs, local glucocorticoids, tumor necrosis factor inhibitors, as well as appropriate exercise programs, physiotherapy, and patient education [14]. Although combined treat- ments are recommended for AS, this study focused on exercise rehabilitation [15]. Researchers have demonstrated that physical activity can decrease pain and disease activity, improve mobility, and increase physical functionality in patients with AS [16,17].

\subsection{Rationale for exercise}

Guidelines for the treatment of AS cannot be applied uniformly; age and the severity of the disease should be considered, particularly with regard to the type and intensity of exercises [18]. The aims of exercise programs are indicated in the long-term goals of patients with AS, including posture, mobility, and respiratory function [19].

Pain and stiffness generally indicate an inflammation of joints and periarticular structures, which, if not mobilized, will lead patients with AS to adopt a comfortable position, commonly with a forward flexed posture [18]. Thus, the focus of exercise should be on maintaining or improving mobility in patients with AS. For patients with AS, mobility is related to relief from pain and stiffness and functional improvements. Regular exercise in patients with AS can prevent a decrease in spinal and hip mobility [20]. Because bony ankylosis can occur in numerous joints around the thorax, resulting in limited movement of the chest wall, breathing exercises are often practiced to maintain or improve the vital capacity and mobility of the thorax [21].

\subsection{Assessment before exercise}

Some physical measurements for patients with AS that have been reported in the literature can be used as a baseline to measure the effects of exercise rehabilitation [18]. There are several valid techniques of measuring spinal and hip mobility [20]. Some of the more important measurements for patients with AS include the Smythe test for thoracolumbar extension and flexion, cervical range of motion, lateral trunk flexion, height, and finger-to-floor distance, as well as the Schober test for lumbosacral flexion. In addition, chest expansion should be monitored, along with regular lung-function tests, owing to the potential for diminished movement in the costovertebral joints and their effect on vital capacity [20].

Outcome parameters for evaluating various aspects of functionality have been developed not only for AS symptoms such as pain and stiffness but also for related domains, such as disease activity, mobility, physical and emotional functioning, quality of life, and social participation [21]. Table 1 shows the domains and instruments in $\mathrm{AS}$ [22]. 
Table 1

Domains and instruments in ankylosing spondylitis [22]

\begin{tabular}{|c|c|}
\hline Domains & Instrument \\
\hline \multicolumn{2}{|l|}{ Single domains } \\
\hline Pain & Visual analogue scale, numeric rating scale \\
\hline Peripheral joints & Number of swollen joints \\
\hline Fatigue & Visual analogue scale, numeric rating scale \\
\hline Global patient assessment & Visual analogue scale \\
\hline \multicolumn{2}{|l|}{ Composite domains } \\
\hline \multirow[t]{2}{*}{ Disease activities } & Bath $\mathrm{AS}^{*}$ disease activity index (BASDAI) \\
\hline & AS* disease activity score (ASDAS) \\
\hline \multirow[t]{2}{*}{ Physical functioning and mobility } & Bath AS* metrology index (BASMI) \\
\hline & Bath $\mathrm{AS}^{*}$ functional index (BASFI) \\
\hline \multirow[t]{2}{*}{ Quality of life and social participation } & Short-form-36 (SF 36) \\
\hline & AS-Quality of Life (AS-Qol) \\
\hline Employment & Work productivity and activity impairment questionnaire (WPAI) \\
\hline
\end{tabular}

$\mathrm{AS}^{*}$ : Ankylosing Spondylitis.

\subsection{Home exercises for patients with AS}

Nolte and Rensburg [19] reported that a pragmatic approach to exercise might be required depending upon the patient's circumstances. According to their study, patients with AS who are employed often cannot afford the time for in-patient treatment. Consequently, inhome exercises can produce improvements equivalent to those seen with in-patient treatment, although the latter is more likely to produce greater improvements in the short term. To improve adherence, home exercises should be tailored to fit into the daily routines. Exercises that can be performed throughout the day are useful, such as corner pectoral stretches, on all trips to the bathroom. Self-monitoring of physical measurements, such as height, can be helpful because patients are often surprised to find out how much height they have lost [19].

\subsection{Intensity, duration, frequency, and type of exercise}

Santos et al. [23] found that patients with AS who exercised approximately two to four hours a week had lower disease activity and improved function compared with those who did not. Their results also indicate that patients who performed intensive daily exercises experienced functionality, but not a decrease in disease activity, which suggests that the health benefits are derived from moderate, consistent exercise. Uhrin et al. [24] searched exercise-based changes in patients with AS in 2000. They revealed that unsupervised recreational exercise and back exercises improved the health status of patients with AS, but that specific benefits varied with the duration of AS. At least 200 minutes per week (>30 min/day) of recreational exercise was associated with decreased pain and stiffness in patients with AS; however, weekly exercise of a lower duration was not.
Back exercises were related to graded decreased pain and functionality for patients with longstanding AS, with the greatest benefits seen in those who performed these exercises at least five days per week. Consequently, it is recommended that patients with AS exercise at least 30 minutes per day and perform back exercises for at least five days per week. Consistency, rather than quantity, appears to be more important. Rouse et al. [25] conducted an open-response survey exploring the perceived effects of physical activity for patients with AS. According to their study, $53 \%$ of patients with AS ( $n=149 ; 60 \%$ female) participated in at least one period (15 min) of strenuous physical activity, $79 \%$ in at least one period of moderate activity, and $93 \%$ in at least one period of mild activity, such as walking (62\%), swimming (22\%), cycling (17\%), filing (15\%), exercise class $(13 \%)$, running $(13 \%)$, gym $(10 \%)$, and yoga $(9 \%)$. Pain and fatigue were the most frequently identified factors that prevented patients with AS from being more physically active. Patients with AS also reported more autonomously driven motivations than controlled motivations to participate in physical activity.

\subsection{Research questions}

This study evaluates home-based rehabilitation in patients with AS during the COVID-19 pandemic. To this end, this study presents the following research questions:

First, what are the essential considerations in homebased rehabilitation for AS patients over 60 years of age during the COVID-19 pandemic?

Second, how can home-based rehabilitation be done for AS patients over 60 years of age during the COVID-19 pandemic? 
Table 2

The demographic characteristics of the expert panel

\begin{tabular}{cclcl}
\hline Expert no. & $\begin{array}{c}\text { Age } \\
\text { (years) }\end{array}$ & Gender & $\begin{array}{c}\text { Career } \\
\text { (years) }\end{array}$ & Occupation \\
\hline 1 & 50 & Male & 22 & Scholar \\
2 & 49 & Female & 17 & Scholar \\
3 & 46 & Male & 16 & Scholar \\
4 & 47 & Female & 17 & Scholar \\
5 & 52 & Male & 23 & Scholar \\
6 & 53 & Male & 24 & Doctor \\
7 & 48 & Female & 19 & Doctor \\
8 & 49 & Male & 20 & Doctor \\
9 & 52 & Male & 21 & Official \\
10 & 51 & Female & 20 & Official \\
\hline
\end{tabular}

\section{Research method}

This study examines home training conditions and challenges for people 60 years of age or older with AS in the COVID-19 era by considering potential qualitative improvements. To this end, a panel of 10 related experts was surveyed, and their responses were analyzed using the Delphi method [26]. The Delphi method is an iterative and anonymous process that is used to achieve consensus on a subject using multiple rounds of discussion with controlled feedback. The Delphi approach has been proven effective in gathering expert opinions on a given issue and allows asynchronous interaction with the expert panel. Experts could complete the surveys whenever it was convenient for them. The Delphi method anonymity aspect allows for unfettered conversation without expert opinions being swayed by others [27]. As this study did not involve human subjects, approval from the institutional review board was not necessary [28].

\subsection{Participants}

The Delphi method has been shown to be valid for gathering experts' opinions on a specific problem [29]. This study invited 10 experts to participate in the research via an e-mail with information about the study. Because defining an expert is subjective, an expert panel does not need to be representative of any particular population [26].

This study selected a panel of participants who had more than 10 years of experience in their field. Baker [30] concluded that researchers must strive for the "best options" when selecting and defending the most appropriate panel of available experts. The panel of experts who participated in the final round comprised five scholars ( $\mathrm{PhD}$ holders), three doctors of state-owned hospital with more than 10 years of experi- ence, and two government officials who had more than two years of experience in exercise policy of more than 2 years and a minimum of 10 years of experience in government.

All 10 experts (100\%) participated in the Delphi process, and they all completed the three surveys. Table 2 shows the demographic characteristics of the experts who participated in the final round.

\subsection{Data collection}

Three Delphi rounds were held over approximately one month, from May 1 to August 31, 2021. Each Delphi round was composed of data collection, data analysis, and controlled feedback [26]. The initial survey was an open questionnaire to derive unbiased opinions from the 10 panelists. The second Delphi round was conducted based on the results of the first questionnaire. The third Delphi round was conducted using fivepoint Likert scale based on the results of the second questionnaire.

The first author was assigned as the facilitator, to organize the expert panel and undertake communication with the panelists. The participating experts were required to assess the importance of each item of the questionnaire. Each expert was allowed to reconsider his or her answers on the questionnaires, after they heard the answers of the other panelists. Table 3 presents the research process using the Delphi method.

\subsection{Data analysis}

In principle, consensus is reached if the coefficient of variation for participating experts is greater than 0 and less than 0.5 ; there is a less-than-satisfactory consensus, and additional investigation may be required if the coefficient is greater than 0.5 , and less than 0.8 ; and consensus is poor and additional investigation is definitely required if the coefficient is greater than 0.8 [31]. In all three rounds of this study, the coefficients derived for all items was below 0.5 , which means that experts reached a strong degree of consensus. The content validity ratio (CVR) proposed by Lawshe [32] was determined by the following formula:

$$
\mathrm{CVR}=\frac{\mathrm{Ne}-\mathrm{N} / 2}{\mathrm{~N} / 2}
$$

where CVR stands for content validity ratio, Ne refers to the number of experts who answered 4-Agree or 5Strongly Agree, and N stands for the total number of panel members. The number of panels determines the final decision to keep the item based on the CVR. The 
Table 3

Research process using the Delphi method

\begin{tabular}{|c|c|c|c|}
\hline Round & Question format & Question content & Analysis method \\
\hline First round & Open questions & $\begin{array}{l}\text { Initial views on home training, including the type, } \\
\text { intensity and frequency- of exercise that subjects should } \\
\text { practice during COVID-19 }\end{array}$ & Content analysis \\
\hline Second round & $\begin{array}{l}\text { Structured five-point } \\
\text { Likert type scale }\end{array}$ & $\begin{array}{l}\text { Importance assessment for specific items in each area } \\
\text { derived from content analysis }\end{array}$ & $\begin{array}{l}\text { Calculation for mean and quadrant } \\
\text { range content feasibility, and CVR* }\end{array}$ \\
\hline Third round & $\begin{array}{l}\text { Structured five-point } \\
\text { Likert type scale }\end{array}$ & $\begin{array}{l}\text { Reassessment of importance and feasibility for each } \\
\text { item }\end{array}$ & $\begin{array}{l}\text { Calculation for mean, standard } \\
\text { deviation, content feasibility, and CVR }\end{array}$ \\
\hline
\end{tabular}

${ }^{*} \mathrm{CVR}=$ Content validity ratio.

Table 4

Minimum value of CVR (content validity ratio), $p=0.05$

\begin{tabular}{cc}
\hline Number of panelists & Minimum value \\
\hline 5 & 0.99 \\
6 & 0.99 \\
7 & 0.99 \\
8 & 0.75 \\
9 & 0.68 \\
10 & 0.62 \\
15 & 0.49 \\
20 & 0.42 \\
30 & 0.33 \\
\hline
\end{tabular}

Source: [32].

guideline for the valid CVR value for the evaluated item to be preserved is shown in Table 4. In this study, the CVR should be higher than 0.62 , since the number of panel members was 10 [33].

\section{Results and discussion}

In this study, items with a CVR ratio of 0.62 or less were excluded from the survey questions, and the contents of each question were ranked according to the mean value; the item results are shown in the this section.

\subsection{First round}

After conducting the first Delphi survey of the expert panel, this study was able to obtain various answers for the following questions: What are the essential considerations in home-based rehabilitation for AS patients over 60 years of age during the COVID-19 pandemic? and How could home-based rehabilitation be done for AS patients over 60 years of age during the COVID-19 pandemic? (Table 5).

Among the experts' answers, the key answers with more than 50\% response frequency are listed in Table 3. The key answers for Question 1 are:

- To reduce pain and maintain joint mobility in AS patients, exercise is crucial during the COVID-19 pandemic (100\%);
- Even if AS patients exercise at home during the COVID-19 pandemic, exercise guidance is needed (100\%);

- It is not advisable for AS patients to visit hospitals or rehabilitation centers and exercise with others at the risk of the COVID-19 infection (90\%);

- Patients with AS need to know what to do and how to do exercise during the COVID-19 pandemic $(80 \%)$

The key answers for Question 2 are:

- For AS patients, warm-up exercises are essential to prevent injury (100\%);

- Mobility exercises are really important for those with AS to do, which depends on the patients with AS (100\%);

- Cardiovascular exercises are important for those with AS (90\%) - Cardiovascular training could be riding a bike, using the cross trainer, going for a walk or going for a swim (80\%);

- Strength training could be recommended for those with AS (80\%);

- Since AS will affect patients' joints, it will be better to protect their joints with strength training $(80 \%)$.

Minority opinions included:

- Any exercises that would put pressure on the spine or hips should be avoided in AS patients (40\%);

- Swimming or cycling will be one of the best exercises for AS patients (40\%);

- It is recommended to exercise using a virtual reality program $(30 \%)$.

Based on these responses, the second Delphi survey questionnaire was created using the five-point Likert scale, as noted in the research methods section.

\subsection{Second and third rounds}

Based on the results of the first Delphi round, the second and third Delphi rounds were conducted after 
Table 5

Panel's key answers to the first Delphi survey

\begin{tabular}{|c|c|c|}
\hline Question & Key answers & Frequency $(\%)$ \\
\hline \multirow{4}{*}{$\begin{array}{l}\text { Question } 1 \\
\text { What are the essential considerations in home- } \\
\text { based rehabilitation for AS patients over } \\
60 \text { years of age during the COVID-19 pandemic? }\end{array}$} & $\begin{array}{l}\text { To reduce pain and maintain joint mobility in AS patients, exercise is } \\
\text { crucial during the COVID- } 19 \text { pandemic }\end{array}$ & 100 \\
\hline & $\begin{array}{l}\text { Even if AS patients exercise at home during the COVID-19 pandemic, } \\
\text { exercise guidance is needed }\end{array}$ & 100 \\
\hline & $\begin{array}{l}\text { It is not advisable for AS patients to visit hospitals or rehabilitation } \\
\text { centers and exercise with others at the risk of the COVID-19 infection }\end{array}$ & 90 \\
\hline & $\begin{array}{l}\text { Patients with AS need to know what to do and how to do exercise } \\
\text { during the COVID-19 pandemic }\end{array}$ & 80 \\
\hline Question 2 & For AS patients, warm-up exercises are essential to prevent injury & 100 \\
\hline $\begin{array}{l}\text { How could home-based rehabilitation be done } \\
\text { for AS patients over } 60 \text { years of age during the }\end{array}$ & $\begin{array}{l}\text { Mobility exercises are really important for those with AS to do, which } \\
\text { depends on the ability of patients with AS }\end{array}$ & 100 \\
\hline \multirow[t]{4}{*}{ COVID-19 pandemic? } & Cardiovascular exercises are important for those with AS & 90 \\
\hline & $\begin{array}{l}\text { Cardiovascular training could be riding a bike, using the cross trainer, } \\
\text { going for a walk or going for a swim }\end{array}$ & 80 \\
\hline & Strength training could be recommended for those with AS & 80 \\
\hline & $\begin{array}{l}\text { Since AS will affect patients' joints, it will be better to protect their } \\
\text { joints with strength training }\end{array}$ & 80 \\
\hline
\end{tabular}

Table 6

Essential considerations in home-based rehabilitation for patients with AS over 60 years of age

\begin{tabular}{|c|c|c|c|c|}
\hline Domains & Content & $\mathrm{M}^{*}$ & $\mathrm{SD}^{* *}$ & $\mathrm{CVR}^{* * *}$ \\
\hline \multirow[t]{4}{*}{ Rehabilitation processes and principles } & Goals for rehabilitation treatment & 5.00 & 0.00 & 1.00 \\
\hline & Presenting a home-based rehabilitation program & 5.00 & 0.00 & 1.00 \\
\hline & Patient's understanding of the program & 4.50 & 0.52 & 1.00 \\
\hline & Identifying the patient's cognitive level & 4.33 & 0.49 & 1.00 \\
\hline \multirow[t]{4}{*}{ Rehabilitation purposes and methods } & Mitigating symptoms of AS & 5.00 & 0.00 & 1.00 \\
\hline & Management with home-based rehabilitation program & 4.83 & 0.39 & 1.00 \\
\hline & Finding the suitable rehabilitation exercise & 4.58 & 0.45 & 1.00 \\
\hline & Preventing injury during the program & 4.42 & 0.51 & 1.00 \\
\hline \multirow[t]{4}{*}{ Rehabilitation content and evaluation } & Gymnastics and stretching-centered exercise & 5.00 & 0.00 & 1.00 \\
\hline & Intensity, frequency, and time of exercise & 5.00 & 0.00 & 1.00 \\
\hline & Consultation on program implementation & 5.00 & 0.00 & 1.00 \\
\hline & Supervision and evaluation by family members & 4.17 & 0.58 & 0.83 \\
\hline
\end{tabular}

$\mathrm{M}^{*}=$ Mean; $\mathrm{SD}^{* *}=$ Standard deviation; $\mathrm{CVR}^{* * *}=$ Content validity ratio.

grouping them into "essential considerations in homebased rehabilitation for AS patients over 65 of ages" or "home-based rehabilitation program for AS patients." In addition, the first round was further subdivided into the following domains: rehabilitation processes and principles; rehabilitation purposes and methods; and rehabilitation content and evaluation (Table 6). The second round was also further subdivided into the following domains: home training exercise type; home training exercise frequency; home training exercise intensity; home training exercise time (Table 7).

\subsubsection{Essential considerations in home-based rehabilitation for AS patients over 65 years of age}

According to the three Delphi results, the detailed responses of the expert panels were largely divided into the rehabilitation process and principles, the purpose and methods of rehabilitation, rehabilitation con- tent, and evaluation areas. The experts' perceptions of detailed items and their importance for each area are shown in Table 6.

The panel of experts unanimously agreed that the processes and principles of rehabilitation were important to selecting clear rehabilitation goals and providing ways for patients to practice the exercise autonomously.

Next, it was agreed that understanding of the rehabilitation treatment process should be identified. Explanations or perceptions expressed by the patients' caregivers or families were excluded, as they have been shown to be of little importance. Panelists unanimously considered the most important factors for home-based rehabilitation to be the treatment of stiff joints and pain relief. Experts next agreed that the exercise program for elderly patients with AS should include selfmanagement skills, with the next-highest priority being to find suitable rehabilitation exercises and prevent injuries during home-based rehabilitation. The panel 
Table 7

Home-based rehabilitation program for patients with AS

\begin{tabular}{llccc}
\hline \multicolumn{1}{c}{ Classification } & \multicolumn{1}{c}{ Content } & $\mathrm{M}^{*}$ & $\mathrm{SD}^{* *}$ & $\mathrm{CVR}^{* * *}$ \\
\hline Home training & Fast walking, gymnastics and stretching & 5.00 & 0.00 & 1.00 \\
Exercise type & Riding a stationary bike & 4.83 & 0.92 & 1.00 \\
& Pilates/yoga & 4.58 & 0.49 & 1.00 \\
Home training & 4 6/week & 5.00 & 0.00 & 1.00 \\
Exercise & 3/week & 4.67 & 0.49 & 1.00 \\
Frequency & Daily & 4.33 & 0.65 & 0.83 \\
Home training & Low-intensity & 5.00 & 0.00 & 1.00 \\
Exercise intensity & Moderate-intensity & 4.83 & 0.34 & 0.83 \\
& High-intensity & 4.67 & 0.49 & 0.67 \\
Home training & 1 hour & 5.00 & 0.00 & 1.000 \\
Exercise time & 30 mins & 4.08 & 0.29 & 0.83 \\
& 90 mins & 4.00 & 0.43 & 0.67 \\
\hline
\end{tabular}

$\mathrm{M}^{*}=$ Mean; $\mathrm{SD}^{* *}=$ Standard deviation; $\mathrm{CVR}^{* * *}=$ Content validity ratio.

unanimously commented on the content and evaluation of the home-based rehabilitation program and, and the need for regular consultation with experts, followed by family supervision and evaluation.

The panel suggested that patients carry out their programs with clear, context-specific rehabilitation goals and that they be provided with exercise programs that they can do alone. The panel agreed that these programs aimed to ease symptoms, such as pain, and stiffness and maintain the range of joint movements. The panelists suggested that exercise prescriptions for patients with AS should focus on gymnastics and stretching. Finally, the panelists indicated that patients with AS should consult with experts regularly to ensure they are performing in-home rehabilitation exercises properly.

\subsubsection{Home-based rehabilitation programs}

In this study, the panel conducted a Delphi survey to identify rehabilitation programs that were recommended for patients with AS over 60 years of age, based on the type, frequency, intensity, and time of exercise rehabilitation during the COVID-19 pandemic.

The panel unanimously recommended that fast walking and stretching are appropriate for a home-based rehabilitation program for patients with AS over 60 . The panel also recommended indoor biking, Pilates, and/or yoga for a home-based rehabilitation program, and unanimously recommended home training exercises for one hour, four to six times a week, including low-intensity weight training.

This study confirmed that the panel agreed with the following proposition: "It is recommended that a lowintensity exercise-based program for an hour, four to six times a week, is necessary for patients with AS over 60 during the COVID-19 pandemic." In the final round, the experts agreed unanimously $(\mathrm{CVR}=1.0)$.

\subsection{Limitations}

This study is not without limitations. We did not directly interview or analyze patients with AS. Since the credibility of the resulting recommendations depends on the rigorous use of the Delphi technique, there is a need for consistency and quality in both the conduct and the reporting of studies, such as guidance on Conducting and REporting of DElphi Studies (CREDES) [40]. Therefore, it is necessary to increase survey participation, followed by a slightly more sophisticated use of the Delphi technique in future studies. Also, further research is needed to determine whether the interruption of this exercise program affects the prognosis of patients with AS.

\section{Conclusions}

Patients with AS usually have symptoms of pain and stiffness in their joints, which should be treated with exercise rehabilitation to maintain and prevent disease progression. This is especially needed during the COVID-19 pandemic, as a discontinuation of exercise rehabilitation may lead to disease flare-ups. Traditionally, AS has been managed with a combination of medication and exercise, with the latter appearing to be more effective for AS than for other types of arthritis [34].

The cornerstone of AS medical management includes therapeutic exercise, with the primary objective being to maintain normal posture and activity. Many kinds of physical therapy have been considered, including supervised individual therapy, unsupervised selfadministered individual exercise, and supervised group therapy [19]. Although these exercise recommendations feature prominently in the relevant clinical guidelines for managing patients with AS [35], the specific infor- 
mation to guide exercise planning is lacking [36]. Most published evidence focuses on mobility exercises, while relatively little attention has been paid to other aspects of exercise programs, such as strengthening, balance, and cardiorespiratory exercise, despite the recognition that AS can affect muscle strength, balance, and cardiorespiratory function [37-39]. Similarly, there is little information about recommended dosages (frequency, intensity, and duration) or patient adherence to recommended programs [36]. Thus, it is necessary to evaluate how exercise therapies should be performed during the COVID-19 pandemic.

This study evaluated home-based rehabilitation for patients with AS over 60, using the Delphi method. During the COVID-19 pandemic, when hospital visits are limited, the experts recommended home-based rehabilitation programs for patients with AS over 60 . Based on the results, this study presents the following conclusions. First, it is suggested that home-based rehabilitation for patients with AS over 60 should carry out the programs they can do alone with clear rehabilitation goals. Second, the rehabilitation programs should aim to ease symptoms, such as pain and stiffness, and maintain the range of joint movement in patients with AS over 60. Third, the appropriate exercise prescriptions and regular consultations should be provided for patients with AS, focusing on gymnastics and stretching at home. Fourth, home exercises such as fast walking, riding a stationary bike, Pilates, and yoga are appropriate for a home-based rehabilitation program in patients with AS over 60. Fifth, patients with AS over 60 should repeat low-intensity exercises for an hour a day, four to six times a week. In conclusion, this study suggests that a low-intensity exercise-based program for an hour, four to six times a week, is necessary for patients with AS over 60 during the COVID-19 pandemic.

\section{Conflict of interest}

None to report.

\section{References}

[1] Coskun Benlidayi I, Kurtaran B, Tirasci E, et al. Coronavirus disease 2019 (COVID-19) in a patient with ankylosing spondylitis treated with secukinumab: A case-based review. Rheumatol Int. 2020; 40: 1707-1716. doi: 10.1007/s00296020-04635-z.

[2] Curtis JR, Xie F, Yang S, et al. Risk for herpes zoster in Tofacitinib-Treated rheumatoid arthritis patients with and without concomitant methotrexate and glucocorticoids. Arthritis Care Res. 2019; 71: 1249-54. doi: 10.1002/acr.23769 pmid: http://www.ncbi.nlm.nih.gov/pubmed/30295428.
[3] Jordan RE, Adab P, Cheng KK. Covid-19: risk factors for severe disease and death. The British Medical Journal (BMJ). 2020. doi: 10.1136/bmj.m1198.

[4] Hartley DM, Perencevich EN. Public health interventions for COVID-19: emerging evidence and implications for an evolving public health crisis. JAMA. 2020. doi: 10.1001/jama.2020. 5910.

[5] Lim W-S, Liang C-K, Assantachai P, et al. COVID-19 and older people in Asia: Asian Working Group for Sarcopenia calls to actions. Geriatr. Gerontol. Int. 2020; 20: 547-558. doi: 10.1111/ggi.13939.

[6] Jaehun J. Preparing for the Coronavirus Disease (COVID-19) Vaccination: Evidence, plans, and implications. Journal of Korean Medical Science. 2021; 36(7): e59. doi: 10.3346/jkms. 2021.36.e59.

[7] Coletto LA, Favalli EG, Caporali R. Psoriasis and psoriatic arthritis: How to manage immunosuppressants in COVID-19 days. Dermatol Ther. 2020; 14: e13415. doi: 10.1111/dth. 13415.

[8] Portnow J, Kline T, Daly MA, Peltier SM, Chin C, Miller JR. Multidisciplinary home rehabilitation: A practical model. Clinics in Geriatric Medicine. 1991; 4: 695-706.

[9] Mehta SP, Roy JS. Systematic review of home physiotherapy after hip fracture surgery. Journal of Rehabilitation Medicine. 2011; 43(6): 477-80.

[10] Argent R, Daly A, Caulfield B. Patient involvement with homebased exercise programs: Can connected health interventions influence adherence? JMIR mHealth and uHealth. 2018; 6(3): e47.

[11] Amin Azhari, Ali Parsa. Covid-19 outbreak highlights: Importance of home-based rehabilitation in orthopedic surgery. Arch Bone Jt Surg. Apr 2020; 8(Suppl 1): 317-318. doi: 10.22038/abjs.2020.47777.2350

[12] Linden SVD, Valkenburg HA, Cats A. Evaluation of diagnostic criteria for ankylosing spondylitis. Arthritis \& Rheumatism. 1984; 27: 361-368. doi: 10.1002/art.1780270401.

[13] Kaprove RE, Hugh Little A, Graham DC, Rosen PS. Ankylosing spondylitis. Arthritis \& Rheumatism. 1980; 23: 57-61. doi: 10.1002/art.1780230110.

[14] Jürgen Braun, Xenofon Baraliakos, Uta Kiltz. Secukinumab (AIN457) in the treatment of ankylosing spondylitis. Expert Opinion on Biological Therapy. 2016; 16: 5, 711-722. doi: 10.1517/14712598.2016.1167183.

[15] Éric Toussirot, Daniel Wendling. Recent progress in ankylosing spondylitis treatment. Expert Opinion on Pharmacotherapy. 2003; 4: 1, 1-12. doi: 10.1517/14656566.4.1.1.

[16] Dagfinrud H, Halvorsen S, Vøllestad NK, et al. Exercise programs in trials for patients with ankylosing spondylitis: Do they really have the potential for effectiveness? Arthritis Care Res. 2011; 63: 597-603.

[17] O'Dwyer T, Rafferty T, O'Shea F, Gissane C, Wilson F. Physical activity guidelines: Is the message getting through to adults with rheumatic conditions? Rheumatology. 2014; 53: 1812-7.

[18] Gall V. Exercise in the spondyloarthropathies. Arthritis Care Res. 1994; 7(4): 215-220.

[19] Nolte Kim, Janse Van Rensburg, Christa D. The role of exercise in the rehabilitation of ankylosing spondylitis. International Sport Med Journal. 2001; 2(4): 1-12.

[20] Walker JM, Helewa A. Physical Therapy in Arthritis. Philadelphia, Pa: WB Saunders Co. 1996.

[21] Fisher LR, Cawley MID, Holgate ST. Relation between chest expansion, pulmonary Function, and exercise tolerance in patients with ankylosing spondylitis. Ann Rheum Dis. 1990; 49: 921-925. 
[22] Kiltz U, M, Braun J. Assessments of functioning in patients with axial spondyloarthritis. J Rheum Dis. 2002; 27: 22-29. doi: 10.4078/jrd.2020.27.1.22.

[23] Santos H, Braphy S, Colin A. Exercise in ankylosing spondylitis: How much is optimum? J Rheumatol. 1998; 25(11): 21562160 .

[24] Uhrin Z, Kuzis S, Word MM. Exercise and changes in health status in patients with ankylosing spondylitis. Arch Intern Med. 2000; 160: 2969-2975.

[25] Peter C Rouse, Martyn Standage, Raj Sengupta. Living with ankylosing spondylitis: An open response survey exploring physical activity experiences. Rheumatology Advances in Practice. 2019; 3(2): rkz016. doi: 10.1093/rap/rkz016.

[26] Okoli C, Pawlowski SD. The Delphi method as a research tool: An example, design considerations and applications. Information \& Management. 2004; 42(1): 15-29.

[27] Steurer J. The Delphi method: An efficient procedure to generate knowledge. Esqueletal Radiología. 2011; 40(8): 959-961. doi: 10.1007/s00256-011-1145-z.

[28] Wesley GB, Pharm D. Working with the institutional review board. American Journal of Health-System Pharmacy. 2009; 66(2): 176-184. doi: 10.2146/ajhp070066

[29] Hsu CC, Sandford BA. The Delphi technique: Making sense of consensus. Practical Assessment, Research \& Evaluation. 2007; 12(10): 1-8.

[30] Baker J, Lovell K, Harris N. How expert are the experts? An exploration of the concept of 'expert' within Delphi panel techniques. Nurse Researcher. 2006; 14(1): 57-70.

[31] Dajani JS, Sincoff MZ, Talley WK. Stability and agreement criteria for the termination of Delphi studies. Technological Forecasting and Social Change. 1979; 13: 83-90.

[32] Lawshe $\mathrm{CH}$. A quantitative approach to content validity. Personnel Psychology. 1975; 28(4): 563-575.

[33] Ayre C, Scally AJ. Critical values for Lawshe's content validity ratio: Revisiting the original methods of calculation. Measure- ment and Evaluation in Counseling and Development. 2014; 47(1): 79-86. doi: 10.1177/0748175613513808.

[34] Millner JR, Barron JS, Beinke KM, Butterworth RH, Chasle BE, Dutton LJ, Lewington MA, Lim EGS, Morley TB, O'Reilly JE, Pickering KA, Winzenberg T, Zochling J. Exercise for ankylosing spondylitis: An evidence-based consensus statement. Seminars in Arthritis and Rheumatism. 2016; 45(4): 411-427.

[35] Ozgocmen S, Akgul O, Altay Z, Altindag O, Baysal O, Calis $\mathrm{M}$, et al. Expert opinion and key recommendations for the physical therapy and rehabilitation of patients with ankylosing spondylitis. Int J Rheum Dis. 2011; 15(3): 229-238.

[36] Dagfinrud H, Halvorsen S, Vollestad NK, Niedermann K, Kvien TK, Hagen KB. Exercise programs in trials for patients with ankylosing spondylitis: Do they really have the potential for effectiveness? Arthritis Care Res. 2011; 63: 597-603.

[37] Sahin N, Ozcan E, Baskent A, Karan A, Ekmeci O, Kasikcioglu E. Isokinetic evaluation of ankle muscle strength and fatigue in patients with ankylosing spondylitis. Eur J Phys Rehabil Med. 2011; 47: 399-405.

[38] Vergara ME, O'Shea FD, Inman RD, Gage WH. Postural control is altered in patients with ankylosing spondylitis. Clin Biomech (Bristol, Avon). 2012; 27: 334-340.

[39] Berdal G, Halvorsen S, van der Heijde D, Mowe M, Dagfinrud $\mathrm{H}$. Restrictive pulmonary function is more prevalent in patients with ankylosing spondylitis than in matched population controls and is associated with impaired spinal mobility: A comparative study. Arthritis Res Ther. 2012; 14: R19.

[40] Jünger S, Payne SA, Brine J, Radbruch L, Brearley SG. Guidance on Conducting and REporting DElphi Studies (CREDES) in palliative care: Recommendations based on a methodological systematic review. Palliat Med. 2017; 31(8): 684-706. doi: 10.1177/0269216317690685. Epub 2017 Feb 13. PMID 28190381. 